\title{
KEYNOTE PAPER: HARDWARE COMPILATION - A NEW TECHNIQUE FOR RAPID PROTOTYPING OF DIGITAL SYSTEMS - APPLIED TO SENSOR VALIDATION
}

\author{
M. P. Henry \\ Department of Engineering Science, Parks Road, Oxford OX1 3PJ
}

\begin{abstract}
This paper provides tutorial introductions to Field-Programmable Gate Arrays (FPGAs) and the concept of hardware compilation - the translation of a high-level programming language directly into a hardware design. As an illustration, a simple stepper motor control program is presented. The research aims of the sensor validation programme are described, and the benefits of using hardware compilation techniques are presented. This leads on in the conclusion of the paper to a more general discussion of the interaction between research and technology, and in particular the influence of information technology upon control engineering.
\end{abstract}

Key Words. Field-Programmable Gate Arrays (FPGAs); Hardware Compilation; Handel; Occam; Transputer; Sensor Validation; Uncertainty; Fault Detection and Diagnosis; Fieldbus.

\section{INTRODUCTION}

Workers in the control domain have traditionally been concerned with the development and applications of algorithms (for example for control or identification). New software technologies (e.g. neural nets, fuzzy logic, or AI techniques) have more recently become a focus of interest as alternative ways of formulating and solving problems which have been difficult using more traditional methods. Whether such software has run on a general-purpose computer or dedicated hardware (e.g. neural net or fuzzy logic ASICs) has usually been of secondary importance.

Developments in hardware have tended to be of less direct interest to control researchers, although in practice they have been of great importance in introducing research ideas into industrial practice. Examples include digital controllers making auto-tuning commonplace, and the more general increase in computational power which has afforded increasing sophistication in all control applications.

This paper describes two new technologies: Field-Programmable Gate Arrays (FPGAs), which are hardware devices, and Hardware Compilation, which is a software technique for configuring FPGAs. In the long term they may have a profound impact on all aspects of data processing, but they are of more immediate value in rapid and reliable prototyping of hardware interfaces, a key issue in many control research projects and applications.
Firstly FPGAs are described along with the conventional processes used to configure them. Next, the motivation behind the concept of hardware compilation is described, and a particular language, Handel, is presented. A simple example of a stepper motor controller is given, for which a complete Handel program is developed. As an example of a control engineering project, the sensor validation programme at Oxford is outlined, together with the projected and actual benefits gained by using hardware compilation techniques. In the conclusion some consideration is given to the interaction between research and technology, and in particular the enormous influence information technology is having upon control engineering.

The paper thus serves a number of purposes. In addition to describing FPGAs and hardware compilation themselves, it is intended to give an example of how the introduction of a new technology, itself the subject of a parallel research program, can be used to assist in the investigation of an entire class of research problems, and bring closer the possibility of generating significant industrial benefit.

\section{FPGAs}

\subsection{Definition}

A Field-Programmable Gate Array is a silicon device containing an array of computing blocks (called Configurable Logic Blocks or CLBs), each of which can perform a limited number of logical operations. An FPGA also contains a number of I/O Blocks (IOBs), providing an interface between each external package 


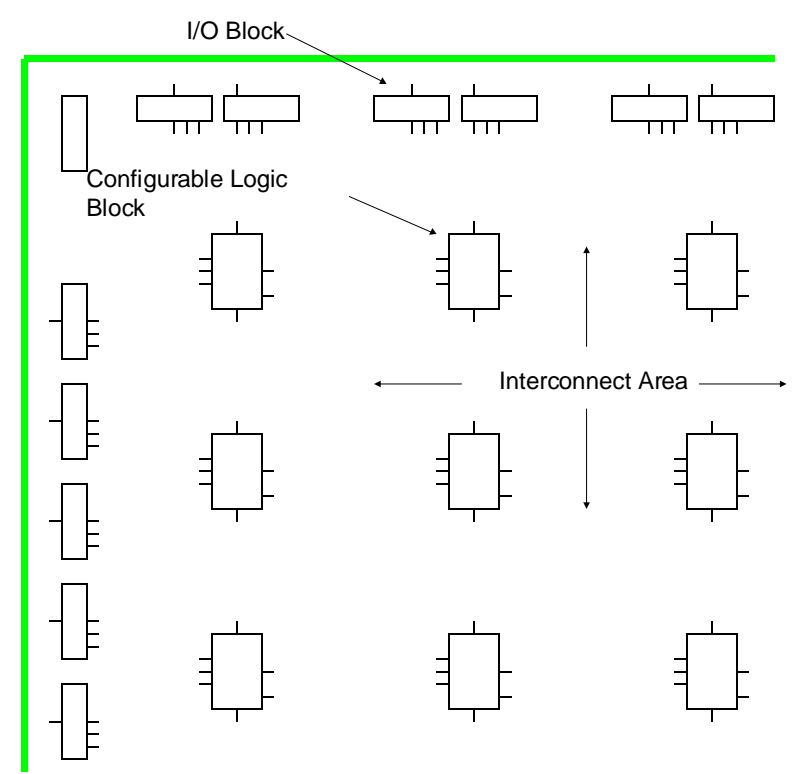

Fig. 1. Structure of Xilinx 2000 series Field-Programmable Gate Array

pin and the internal logic. The exact function of each block, and the interconnections between them, are determined by on-chip static memory, which is configurable. Typically the FPGA is configured once on powerup from an EPROM or a processor, but it is also possible to repeatedly reconfigure the FPGA to perform a series of different functions.

The chief attraction of the FPGA is that its functionality is defined by software, and so redesign merely entails reprogramming, which can take place within milliseconds. During the development of a product requiring a moderate level of processing logic (for example a networking card), the component layout and hardwired configuration can be settled early on, leaving the detailed functionality to be developed, debugged and refined within the FPGA. More radical design changes can also be envisaged, where for example a different communication protocol can be implemented using the same hardware but a different FPGA configuration.

Economically, FPGAs are attractive in a variety of development applications. Characteristics of suitable projects might include the following:

- the expense or complexity of a microprocessor design is not warranted;

- a microprocessor design would be too slow;

- the market volume does not justify the high cost and extensive lead time of ASIC design;

- the development entails the rapid prototyping of an ASIC or microprocessor;

- the development requires extensive interfacing between complex components;

- the development entails on-line reconfiguration of hardware.

Their flexibility allows suppliers to respond rapidly to developments in technology-driven markets. Their use is growing rapidly: one estimate suggests the programmable logic segment could grow to $25 \%$ of the total CMOS logic market by the year 2000 (Vonderschmitt 1994).

\subsection{The Xilinx 2000 series FPGAs}

A typical example of an FPGA family is the Xilinx 2000 series (Xilinx, 1994), the architecture of which is shown in Fig. 1. The XC2064 provides an array of $64(8 \times 8)$ logic blocks and $58 \mathrm{IOBs}$, equivalent to approximately 1200 logic gates, while the XC2018 provides 100 logic blocks and 74 IOBs corresponding to some 1800 gates. Configuration program sizes are $12 \mathrm{~K}$ bits and $17 \mathrm{~K}$ bits respectively. Logic gate toggle rates vary from 33$130 \mathrm{MHz}$. The maximum clock frequency that can be supplied to the FPGA depends upon the particular design, which dictates the propagation delay through connected gates.

Each IOB may be programmed to provide input or output compatible with either TTL or CMOS. Each CLB (Fig. 2) consists of a combinatorial logic section, a storage element (a D-type flip-flop), and an internal routing section which controls each of the multiplexors. There are four inputs to each block labelled A, B, C and $\mathrm{D}$; the flip-flop output $\mathrm{Q}$ can also be used as an input to the combinatorial section. The logic can perform a single operation on four inputs (in which case internal values $F$ and $G$ are identical) or two independent operations on three.

For example, the logic section may be configured to calculate the function $((\mathrm{A} \wedge \mathrm{B}) \vee(\neg \mathrm{C} \wedge \mathrm{D}))$, or the two functions $(\mathrm{A} \wedge \mathrm{C} \wedge \mathrm{Q})$ and $(\mathrm{B} \wedge \mathrm{C} \wedge \neg \mathrm{Q})$. The two CLB outputs X and Y may be set to the output(s) of the logic operation(s) and/or Q, and may be used to provide inputs to other logic blocks or outputs via IOBs.

Blocks may be connected in a number of ways:

- adjacent blocks can be linked by localised direct connection.

- 'long lines' distribute signals that must travel a

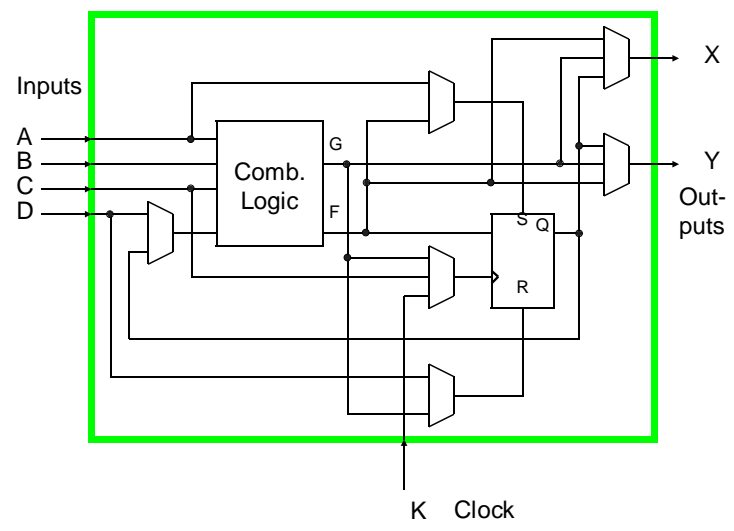

Fig. 2. Configurable logic block (Xilinx 2000 series) 
long distance or must have minimum propagation delay among multiple destinations (normally down a column of blocks).

- General purpose interconnect provides a method of connecting arbitrary blocks in the array.

The 2000 series is only one of several commercial FPGA architectures. Xilinx themselves have released the 3000 and 4000 series, each of which have higher block counts and functionality per block (Table 1); other manufacturers include Lattice (1994), Atmel (1993) and Altera (1993).

Table 1: Three Generations of FPGAs

\begin{tabular}{lrrr}
\hline & XC4025 & XC3090 & XC2018 \\
\hline Year released & 1992 & 1989 & 1985 \\
Gates (approx) & 20,000 & 10,000 & 1,800 \\
Flip-flops & 2,560 & 928 & 174 \\
IOBs & 256 & 144 & 74 \\
CLBs & 1,024 & 320 & 64 \\
CLB logic inputs & 9 & 5 & 4 \\
CLB logic outputs & 4 & 2 & 2 \\
\hline
\end{tabular}

Continued improvements in architectural and manufacturing process technology suggest compound annual growth rates in speed, component density and performance/price ratio of $25 \%, 58 \%$ and $30 \%$ respectively. The state-of-the-art FPGA in the year 2000 is projected to have 150,000 usable gates and a gate toggle rate of 250MHz (Vonderschmitt 1994).

\subsection{Design Techniques}

The above description has demonstrated both the enormous flexibility of the FPGA and also the extremely fine granularity with which its functionality is defined. With regards to the former, a $17 \mathrm{Kbit}$ configuration program implies $2^{17 \mathrm{~K}}\left(\approx 10^{5240}\right.$ ) possible configurations; the latter implies that design by direct manipulation of the function of each CLB and IOB is likely to be a slow and painful process for all but the most trivial of projects. In fact an editor is often provided for modifications at the lowest level, but in practice higherlevel design tools are almost invariably used; these subsequently map onto an FPGA configuration.

A number of design tools may be used to configure the Xilinx FPGA, as illustrated in Fig. 3. Similar routes are used for the devices of other manufacturers. A single path follows from the Xilinx Netlist Format (XNF) file through to the final bit-stream used to configure the FPGA (Xilinx, 1993). XNF is an intermediate design description which defines pins, signals and logic gates, without specifying which function is carried out by which block, or indeed which particular Xilinx device is to be used. A large number of third-party design tools may be used to generate XNF format files.
$\mathrm{XNF}$ has the equivalent role of intermediate code in conventional software compilers (Aho and Ullman, 1977), and represents a well-known economy. A variety of high level design techniques (languages) can be used to generate a single intermediary description (perhaps three-address code), which is then further translated into a form appropriate for the particular FPGA (computer) hardware. Given $n$ high-level tools and $m$ hardware platforms, only $m+n$ translation programs are required for complete interchangeability; without the intermediary form $m \times n$ programs are needed.

FPGA design tools can be largely divided into two categories - graphical and textual. A third type, the hardware compiler, will be described in more detail later.

Schematic Drawing Packages. A natural method of specifying an electronic design is through a drawing package. Typically, graphical symbols are used to represent simple devices such as AND gates, or more complex elements such as multiplexers or dividers. Symbols are instantiated, configured and connected on a computer screen using mouse and keyboard, typically with little consideration of the final hardware implementation (e.g., ASIC, FPGA or surface-mounted components on a PCB). User-defined macros or modules allow for maximised re-use of design effort. A translation facility may then be used to convert a drawing into an XNF file. Examples of drawing packages include Dash (FutureNet, 1987), Schema (Omation, 1988) and Workview (Viewlogic, 1991).

Behavioural Description Packages. An alternative to graphical design is to specify requirements using a variety of textual forms, such as declarations (e.g., pin

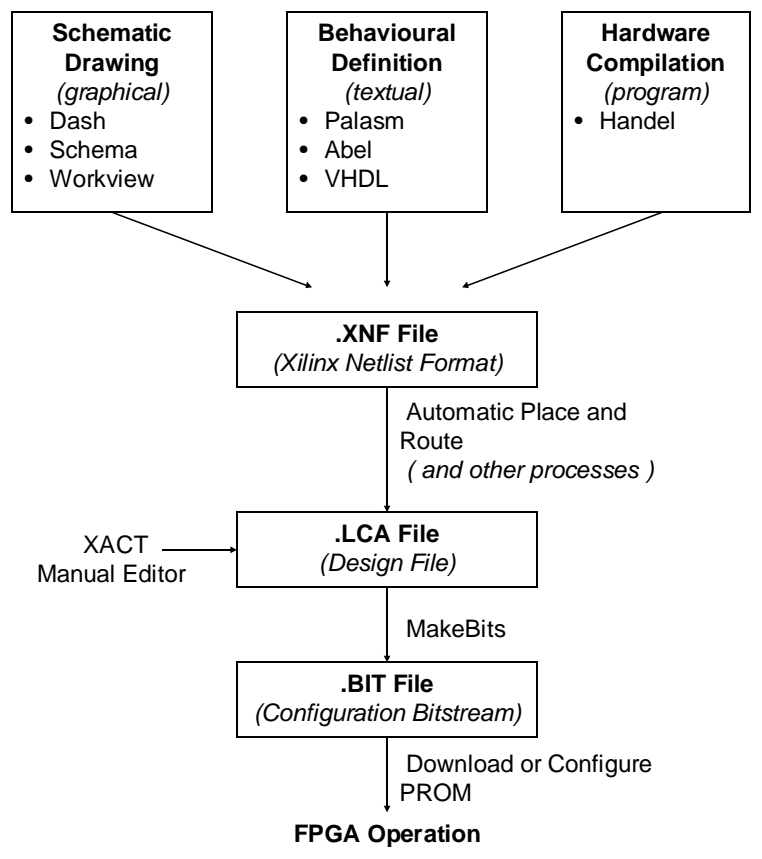

Fig. 3. FPGA design path (Xilinx devices) 
assignments), Boolean equations, truth tables, and state-machine descriptions. Note that a description language may itself be the output from a graphical tool or other design process. Again, a high-level description can be implemented in a wide range of technologies through the use of appropriate conversion utilities. Typical examples are Palasm (AMD, 1991) and Abel (Data I/O, 1993).

Special mention must be made of VHDL (IEEE, 1994), which is the VHSIC Hardware Description Language, where VHSIC is a Very High Speed Integrated Circuit. This is a standardisation initiative begun by the US Defence Department. It aims at providing a language for describing a wide range of electronic hardware, with the key objectives of working identically on any simulator and being entirely independent of hardware technology or design methodology. This standard is now being managed and promoted by the IEEE.

Automatic Placement and Routing. The key stage in the transformation of an XNF file into a configuration bit stream is Automatic Placement and Routing (APR). This process converts a netlist into the complete configuration of the selected FPGA architecture (an LCA file). Functions are placed into specific CLBs, IOBs are assigned specific roles, and the interconnections between the blocks are routed.

APR is a complex, constrained optimisation problem. As pointed out earlier, even the simplest devices can be configured in an enormous number of different ways; with APR both the placing and then the routing of a particular design are likely to have astronomical numbers of potential solutions. There are, however, constraints: the number and layout of the CLBs and IOBs, the limited resources for providing interconnections between them, and specific signals which must be assigned to particular IOBs due to interfacing requirements with the external system. A simulated annealing technique is used to find an 'optimum' placing. The cost function used is a formula which includes the length of the routes (affecting propagation delay), the number of available routing channels and user-defined weightings; the latter may be used to minimise propagation delays in key parts of the design.

APR is computationally highly expensive. A large design using 3000 series devices may take several hours to place and route on a Sparc workstation. When the proportion of FPGA resources required is high (say $85 \%$ ), the software may not be able to find any solution. There are a number of ways of simplifying the problem. A larger FPGA may be used, thus increasing the degrees of freedom. Constraints may be removed (e.g. that a particular IOB should perform a particular function). Finally, parts of the design can be removed from the APR problem, and then hand-crafted into the resulting solution using the manual editor.
Execution Errors. Even when a design is successfully completed, there is no guarantee that a fault-free performance will result. The principal causes of problems are the use of asynchronous logic (which may cause occasional, disruptive glitches) and designs where the propagation delays are excessive. In the latter case the gating from the previous clock cycle may not have been completed before the next clock cycle begins. In general there is a maximum clock rate at which an FPGA configuration will operate successfully, which is likely to be considerably slower than the nominal gating frequency.

\section{HARDWARE COMPILATION}

At a first glance, the concept of a hardware compiler that is, an imperative programming language which is compiled directly into an XNF file - may seem to be just another form of descriptive design tool, like VHDL. An examination of the motivation behind the hardware compiler described in this paper, called Handel, reveals it to be an initiative based on fundamental research into design methodology.

\subsection{Correctness in Design.}

Hoare and Page (1994) consider complex design tasks (for example the implementation of a real-time process control system), which are typically decomposed into a progression of design phases. Usually the end-product of each phase is a 'document' (human- and/or machinereadable), which is a less abstract, more detailed specification than its predecessor, and which forms the starting point of the next design phase. Thus the design chain begins with the most abstract statement of design requirements, and ends with the final plant, hardware, and, where software is involved, machine code running on one or more processors.

The FPGA design paths described in the previous section (Fig. 3) illustrate perhaps the last few stages of such a design process. Each file format constitutes a 'document', and a design progresses from one of a variety of possible 'high-level' description formats through to the final bit pattern to be loaded onto a particular FPGA.

In reality, the design process for non-trivial projects is rarely smooth. A design stage may introduce errors in its transformation from one document to the next; alternatively the language in which a document is written may be unclear or ambiguous. The latter possibility is particularly likely in large engineering projects, where a great diversity of skills is needed. Even when each design phase is carried out with meticulous care, misunderstandings may easily arise between different specialists, and as a result 'bugs ... (may) congregate and breed in the interfaces between one phase and the next'. 
Hoare and Page state that improving the intrinsic reliability of the design process is a valid and valuable topic of engineering research, and they argue that the only viable approach is to introduce mathematical formality both in the documents themselves (to ensure that they are well-defined) and in the transformation (i.e. design activity) at each stage. They point out that all that is necessary to prove the correctness of each design stage is to demonstrate that the output document $\left(D_{k+1}\right) \log i$ cally implies the input document $\left(D_{k}\right)$. For the implication relation is transitive, and so if

$$
D_{k-1} \Leftarrow D_{k} \text { and } D_{k} \Leftarrow D_{k+1} \text { then } D_{k-1} \Leftarrow D_{k+1}
$$

In other words, if $D_{k}$ is implemented by $D_{k+1}$, and $D_{k-1}$ by $D_{k}$, then $D_{k-1}$ is implemented by $D_{k+1}$, and hence by transitivity so is $D_{0}$, the original design specification.

Such a simple notation belies the ambition of this aspiration, which is being actively pursued in a whole range of research topics collectively termed 'formal methods'. There are two principal tasks. Descriptive languages must be created which have well-defined semantics and which are adequately expressive for the domains of interest. Transformations (usually algebraic) must be developed which prove an equivalence or implication relation between expressions within a single language or in two different languages.

Considerable research effort has delivered a much greater understanding of what constitutes a 'good' (i.e. semantically unambiguous and usefully expressive) language, and the conditions under which proofs of correctness are possible. However, thus far 'success' has been only partial: total design correctness may be proved in certain (typically simple) cases, but the tools which have been developed as a consequence of the research can prove at least partial correctness when applied to many practical problems. These tools include CSP (Hoare, 1985), a theoretical model of distributed communication, Z (Abrial, 1985), a specification language, the computer language occam (Jones and Goldsmith, 1988; Inmos, 1988a) and the transputer processor (Inmos, 1988b).

occam and the transputer are perhaps the most concrete examples (from an engineering perspective) of these developments. In occam, an application is described as a number of concurrent processes communicating via channels. The architecture of the transputer reflects this model. A key attraction is that occam has a clear semantics: a piece of occam code has a precise meaning; the behaviour of a transputer executing the code is entirely predictable. occam is thus an ideal language in which to specify designs (as the initial specification, the final implementation, or any step in between) and to apply formal proofs and transformation techniques. This contrasts with conventional design languages such as C (or indeed VHDL) which do not have well-defined semantics. For example, in $\mathrm{C}$, the behaviour of certain program constructs are 'undefined' or 'implementation-specific'. Without a clear semantics, of course, formal proofs are rendered impossible.

Given that complete formal proof tools are still not available and applicable for almost all design domains, why should these developments be of immediate interest to the practising control engineer? Existing tools have been designed so as to eliminate the possibility of entire classes of error; as formal methods research continues these tools will be further enhanced. Over time, designs using these tools may become 'asymptotically correct'; in the meantime they are merely extremely reliable. The practical implications for a design process are hopefully shorter test and refinement (i.e., debugging) timescales and a more reliable end product.

Such benefits are reduced if the tools themselves cannot easily be used by design engineers. While changes in design thinking and practice are to be expected, some of the early tools have provided rigour at the expense of ease of use. The importance of human productivity has always been recognised, and subsequent enhancements (e.g. the introduction of data types in occam2 (Jones and Goldsmith,1988)) have provided additional features of benefit to the user without sacrificing semantic rigour.

\subsection{Merging of Hardware and Software}

Given the enormous range of design activities the human species is engaged in, why should FPGA configuration attract early interest? The driving force is a vision of a new type of computer architecture incorporating configurable logic: if computer code can be transformed into hardware, then the distinction between the concepts of hardware and software blurs.

Hoare and Page (1994) describe an experimental board called HARP, consisting of a transputer with a large FPGA acting as a co-processor. A simplified diagram of the system is shown in Fig. 4. Both the transputer and FPGA have local memory, and they share a 32-bit bus, which enables the transputer to download configuration data to the FPGA. The transputer controls the clock so that it generates a frequency suitable for the current FPGA configuration. A given application can be split into occam code running on the transputer and Handel code implemented in the FPGA. Tools are not yet available for the partitioning process to use a single source file for both hardware and software. However, initial results using the HARP board are encouraging: for example a spell-checking algorithm ran 25 times faster on the co-processor than on the transputer.

The HARP board is seen as a prototype of a hybrid processor containing significant proportions of configurable hardware. Hoare and Page project a vision of 


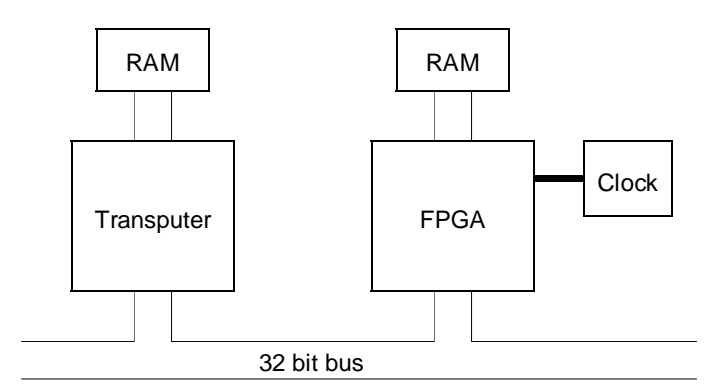

Fig. 4. Simplified architecture of the HARP board

computing technology where the user is unaware of whether his/her processes are being executed in hardware or software, and where the available configurable logic is reprogrammed on the fly to deal with the most intensive demands on the system. Active application areas for these concepts include vision, multi-media and speech processing.

These developments are in themselves clearly highly desirable, leading to further considerable increases in available computational power, improved software reliability and increased design productivity. However, there is another major application area which is perhaps of more direct interest to control engineers. Page (1993) describes an application where Handel is used to specify and configure a hardware interface between a set of sensors and a control computer in a robotics application. Further discussion of the potential use of hardware compilation techniques for interface design in control applications is deferred to a later section of the paper.

\subsection{Handel for FPGA design}

Handel is an occam-like language; the hardware compiler converts a Handel program into an XNF file.

Even accepting the desirability of proving design correctness (or indeed hybrid hardware/software systems), it is legitimate to ask whether an imperative language is appropriate for specifying an FPGA configuration for conventional design problems. Two key issues are whether a program paradigm results in an efficient FPGA configuration, and whether the language is sufficiently expressive to allow a human or automated designer sufficiently free (and, in the human case, intuitive) description of the desired result.

Efficiency. At first sight the efficiency issue might appear to be a considerable obstacle. A programming language is normally intended to be executed on a processor, and hence assumes the availability of such facilities as an instruction set, RAM for code and data storage, and a fetch-execute cycle. None of these is provided in a simple array of configurable logic.

Of course it is possible to configure an FPGA to act as a processor, with its own instruction set and other facilities; this is an active research topic in itself (Page,
1994) with obvious applications for prototyping new processors. However, this approach is hardly suitable for all FPGA applications, as the silicon overhead to implement a processor and memory is considerable.

Handel does not attempt to emulate a processor. Program variables are stored in flip-flop registers, while expressions are implemented as circuitry to modify register contents. It is straightforward to implement these efficiently. More challenging is the implementation of circuitry to control program flow. The 'one-hot' approach, in which a flip-flop is used to control the execution of each program statement, has been shown to be particularly space-efficient on Xilinx FPGAs (Knapp 1990; Schlag et al. 1990). Page and Luk (1991) describe how the various Handel programming constructs are implemented in an FPGA configuration.

Expressive Power. A second concern is how expressive Handel is, in other words how easily and naturally it allows the designer to state his/her requirements for FPGA functionality. This raises another potential discrepancy between the program paradigm and FPGA circuitry. Most programming languages prescribe sequential steps, while FPGA operations are largely (and optimally) carried out in parallel. It is here that Handel's occam-like ability to describe parallel processing requirements proves highly effective.

Another issue concerns the relative merits of textual as opposed to graphical design. Most commercial design tools use a graphical interface, even though almost all engineers are now familiar with programming, and evidently one form may be mapped into the other. It may be argued that a textual form more naturally expresses iteration and parameterisation, the latter being particularly important for re-use in design. A textual form may also be generated and edited independently of the associated design tool (in particular by another program). Finally in the author's experience, once it has been demonstrated that a program-style definition is successfully mapped into the required gates and routes, the more abstract description is readily accepted, and the more detailed implementation (which may be displayed graphically) is of little interest.

Notwithstanding the above, Handel is a new language, and experience in its usage is being acquired in a number of application areas. On the basis of this experience additional features are being incorporated to ameliorate difficulties faced by users in defining, and obtaining, their FPGA design requirements.

Correctness. A third issue is the extent to which Handel embodies the design methodology outlined earlier. Handel itself is essentially a sub-set of occam and as such inherits much of its denotional semantics. Therefore it is known precisely what every program written in Handel means. In addition, it inherits occam's 
algebraic semantics. There are thus algebraic laws for transforming a Handel program into its proven-equivalent normal form (Hoare and Page, 1994).

However, unsurprisingly, XNF does not have a rigorously defined denotional semantics. It is therefore not possible to formally prove the transformations from Handel normal forms into a netlist. Overall, therefore, the hardware compilation technique as currently implemented can best be described as extremely reliable but not proven correct.

In any case, the place and routing technique (Section 2.2) used to transform the netlist into the final configuration is certainly unlikely to yield to a formal analysis. One can only have a moderate confidence that the netlist has been correctly represented in the final FPGA configuration. Here 'moderate' is taken to mean a level of confidence appropriate for today's commerciallyavailable program carrying out a comparably complex task. In the author's own experience the commercial place and routing program has not always provided acceptable results.

Of course, there is no fundamental reason why one particular FPGA architecture should be used as the target for hardware compilation. It is certainly reasonable to envisage the development of a netlist language with a well-defined semantics, a corresponding FPGA architecture, and a place and routing algorithm which rigorously transforms one to the other. Such a scheme might prove to be inefficient in comparison with stateof-the-art logic density (Section 2.1), but this would be more than compensated for by the correctness of the final design. It would then be possible to envisage, after further considerable research effort, a design route with proven correct (or at least asymptotically correct) transformations from Handel through to an FPGA configuration. A new FPGA architecture (Oxford Parallel, 1994) represents a first step in this direction.

\subsection{The Handel Language}

A complete description of the language is given by Spivey and Page (1993). This section merely provides an overview of its features sufficient for an appreciation of the programming style, and so that the stepper motor example may be understood.

Handel and SML. The Handel compiler has been implemented within the Standard ML (SML) environment (Milner et al.,1990), rather than as a standalone program. SML provides powerful facilities for automated program generation, and allows easy modification and extension of the language features. A Handel program takes the form of a set of SML expressions, with the following structure:

$<$ declarations $>$

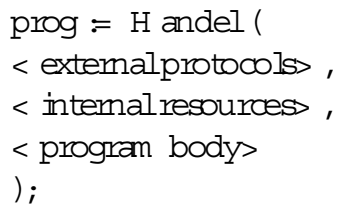

The declarations typically define variables or constants to be used in the program, as described below. The external protocols define how program channels are to interface to the outside world, while the internal resources states which of the currently-defined SML symbols are to be used in the program itself. Both of these are illustrated in the stepper motor example. Finally, the program body defines the set of actions to be taken.

Variables. The data types presently implemented are the channel and the integer. As a variable will ultimately be represented by a hardware register, its bit length must be declared. For example

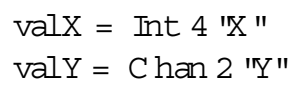

declares two variables $\mathrm{X}$ and $\mathrm{Y}$, of types integer and channel, with bit lengths of 4 and 2 respectively. The string at the end of each declaration is used by the compiler for error reporting and program simulation.

Expressions. A wide range of operators are provided, including unary (e.g. bitwise negation), arithmetic (e.g. +, -, *), comparison (e.g. = = equality, != inequality) and bit manipulation (e.g. ${ }^{\wedge}$ bit-string concatenation, X B T 2 gives the second bit in X). It is a requirement that operands have compatible bit lengths. Constants are indicated by the symbol C:

$$
(P * Q)+C 7
$$

Note that for compatibility, if $\mathrm{P}$ and Q are both 4 bits long then the constant should be 8 bits long. Normally the compiler can deduce the required bit length of a constant, but it is possible to specify the length explicitly using the form $\mathrm{C}$ onst $(7,8)$ (i.e., the value 7 represented as an 8-bit quantity).

Command Sequence. Conventional programming languages are in the main restricted to purely sequential execution. Handel uses a syntax similar to occam's for expressing explicitly whether a list of program statements are to be executed in series or in parallel. For example:

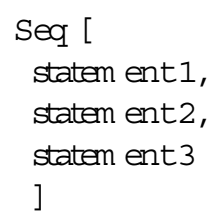


is executed in strict sequence. The first statement is executed immediately, the second statement commences once the first has been completed, and the entire sequence is completed once the final statement has been executed. The statement

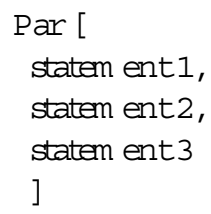

operates differently. Each of the statements commences execution simultaneously, and the entire parallel command is completed once all the individual statements have terminated. As the statements are finally implemented into FPGA circuitry, their execution will be genuinely in parallel. Of course, Parand Seq statements can be nested, and it is these program structures above all others which give occam-like languages their distinctive form. Note that a shorthand form of each is available:

$$
\text { s1 || } 22|| s 3
$$

and

$$
\text { S1 \$\$ S2 \$ S3 }
$$

denote parallel and sequential composition respectively.

Assignment. In Handel assignment uses the familiar notation

$$
\mathrm{X}=\mathrm{Y}+\mathrm{C} 1
$$

but a multiple-assignment statement is also provided:

$$
[\mathrm{X} 1, \mathrm{X} 2, \mathrm{X} 3]=[\mathrm{z} 1, \mathrm{z} 2, \mathrm{z} 3]
$$

is semantically equivalent to

$$
\mathrm{X} 1=\mathrm{z} 1|| \mathrm{X} 2:=\mathrm{z} 2|| \mathrm{X} 3=\mathrm{z} 3
$$

Conditional. This takes the form

$$
\text { If (cond, sl , s2) }
$$

where cond is a Boolean (i.e. one-bit) expression, and sl and $s 2$ are arbitrary statements, executed if cond is true or false respectively. Note that $s 2$, the 'else' clause, must always be provided.

Loops. Two forms are provided:

W hile (cond, s)

U ntil (cond, s)
W hile tests the condition cond at the start of the loop, so the statement smay not be executed. W hile terminates when cond is false. $U$ ntilexecutes sbefore testing cond and terminates when cond is true.

Channel communication. The channel is an important concept in the CSP/occam philosophy. It is the primary means of communication between parallel processes and provides mechanisms for ensuring proper synchronisation. Essentially, a process wishing to input (or output) from a channel must wait until another process wishes to output (or input) from that channel. Data is exchanged, and on completion of the transfer both processes are able to continue execution. In Handel, channels may be used for communication between parallel processes within a single program, or via the FPGA pins to the outside world using the various protocols provided. The notations for input and output respectively are

$$
\begin{aligned}
& \text { chan ?? var } \\
& \text { chan !! exp }
\end{aligned}
$$

where chan is the name of a channel variable. Note that input data must immediately be transferred into an integer variable var, while the result of an expression (of suitable bit length) may be output directly through a channel.

Other language features. The Skip and D elay commands do nothing and then terminate immediately and after one clock cycle respectively. Skip is frequently used to provide a null statement (for example as the else clause in the ifcommand). D elay may be used in a loop body to ensure that some time elapses before the conditional expression is re-evaluated.

Other features include a C ase statement and facilities for creating on-chip ROM and RAM.

Timing Issues. In Handel timing issues are kept as simple as possible. All actions are synchronised to take place on the rising edge of the clock signal, and all expressions evaluate within a single clock cycle. Assignment, D elay, and channel communication (when ready) take precisely one cycle to complete, while all other program constructs require no additional clock cycles. As a consequence, it is possible to predict exactly the number of clock cycles needed to execute a given piece of code.

Handel Commands. Presently Handel is available on Sparc Workstations. The Unix command

\section{\$ handel}

invokes the SML environment and loads definitions related to Handel. As described by Spivey and Page (1993), facilities are provided for loading Handel files, 
compilation with or without an optimiser, and simulation of program behaviour. If compilation is successful, a netlist file is created, to be used as the starting point for the Xilinx commercial place and route software.

\subsection{Example: A Stepper Motor Controller}

This example demonstrates the use of the Handel language by developing a complete program to solve a simple problem. Note that the program developed here is not particularly efficient, nor does it follow 'best practice' with regard to style. The prime intention is to present a clear example to those unfamiliar with an occam-like programming language.

Stepper motors. A stepper motor allows accurate control of the speed and/or position of its spindle, by moving in discrete steps of perhaps a few degrees. Stepper motors are used widely in automated manufacturing and in robotics. A simplified diagram of a typical commercial device is shown in Fig. 5. It consists of 4 d.c. coils and a permanent magnet rotor, with a step size of 1.8 degrees to an accuracy of 5.4 minutes (errors are not cumulative). A residual torque is provided even when the motor is stationary to ensure positional integrity. Motion is controlled by energising the coils in a fixed sequence, as shown in Table 2:

Table 2: Stepper Motor Control Sequence

\begin{tabular}{ccccc}
\hline Step & Q1 & Q2 & Q3 & Q4 \\
\hline $0(4)$ & OFF & ON & ON & OFF \\
1 & OFF & OFF & ON & ON \\
2 & ON & OFF & OFF & ON \\
3 & ON & ON & OFF & OFF \\
4 & OFF & ON & ON & OFF \\
$5(1)$ & OFF & OFF & ON & ON \\
\hline & & & &
\end{tabular} \mid

Each step moves the rotor 1.8 degrees in the appropriate direction, and the motor can operate at a maximum of about 800 steps per second. It is also possible to operate the motor in half-step mode, in which a sequence of 8 control steps are required, each of which rotates the motor by 0.9 degrees.

Problem Statement. The demonstration system used to illustrate hardware compilation techniques is based upon a student laboratory exercise which introduces FPGA concepts and conventional FPGA design techniques. A preconstructed system is provided, as illustrated in Fig. 6.

Two push-buttons are labelled Go and Change_Dir. These two inputs are provided on pins 59 and 56 of the Xilinx 2018 FPGA. The outputs (pins 81, 83, 79 and 82 ) control the stepper motor inputs Q1-4. Some simple

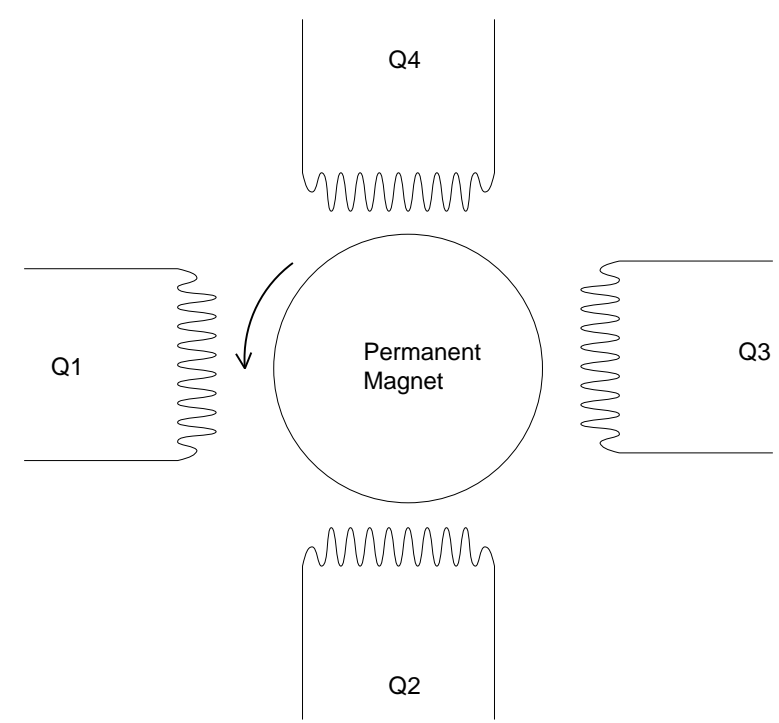

Fig. 5. Simplified stepper motor design

signal conditioning is used to ensure appropriate voltage and current levels for all system components. Finally, a low frequency $(\sim 50 \mathrm{~Hz})$ external clock signal is provided to pin 13 of the chip. This ensures that even if the FPGA updates its output every cycle, the motor will be able to keep up.

The exercise is simply stated: create an FPGA configuration such that the Go and Change_Dir buttons have the following effects on the stepper motor:

- Pressing Go should cause the motor to rotate. The motor should halt as soon as the button is released.

- Pressing Change_Dir should cause the direction of rotation to be reversed. The button should be released before it can cause another change in direction, to prevent threshing.

Problem Analysis. In any strictly sequential programming language, expressing a solution to the problem would be trivial. With the availability of parallel programming constructs, some interest remains. Typically the efficiency of a program is judged by its speed (in the average or worst case) or size. When truly parallel computation is possible, an extra dimension of the design problem is created by considering which steps can be performed in parallel in order to minimise program execution time while retaining semantic integrity.

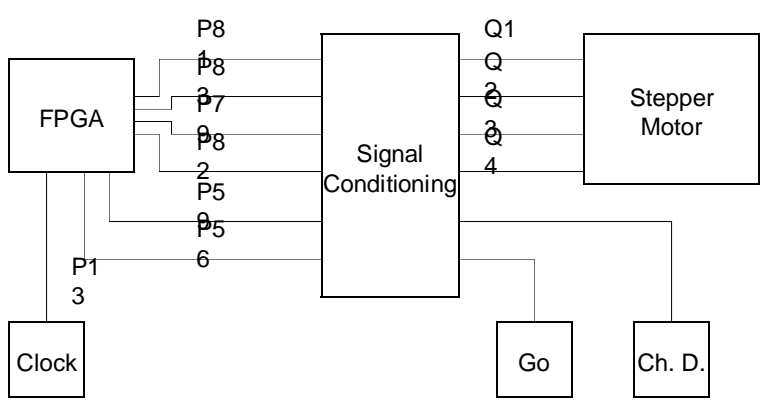

Fig. 6. Stepper motor demonstration system 
In any program a number of constraints operate to restrict parallel processing:

- Certain operations require non-zero periods of time to complete.

- An operation may not be carried out until all of its operands are available.

- It must be impossible for a variable to be simultaneously read and written, or written to more than once, by code executed in parallel.

The last point is vital to ensure semantic integrity. In occam itself, statements executed within each Parare checked by the compiler. Within the Para variable may be written to at most once; it may be read many times, but only if it is not written to. Inter-process communication is dealt with by the channel mechanism which ensures that integrity is maintained.

Handel has slightly more relaxed rules. The timing mechanism within the FPGA hardware is configured so that for each variable a single write and multiple reads can validly take place during each clock cycle.

On the basis of the above restrictions, a program has been drawn up with three processes operating in parallel, as illustrated in Fig. 7. The direction control process reads the Change_Dir signal and determines the current direction. This is used by the position control, together with the Go signal, to determine the current position. Finally, the output generation process uses the current position to construct the output signal Q.

Although the program design envisages three separate 'processes', communication between them is implemented using shared variables rather than channels. This breaks the spirit of the occam philosophy but does not break Handel's integrity rules, and in this case leads to simpler code with fewer variables.

Program Listing. A complete listing of the SML definitions and Handel program is given in Appendix 1. It is stressed that the data provided is complete; nothing further is required to configure the FPGA other than the Handel compiler and Xilinx's standard commercial software. For the purposes of this paper line numbers have been added; of course these are not used (or provided) by Handel itself.

Lines 6-10 provide architectural information, including the chip type (XC2018 with 84 pins and a maximum

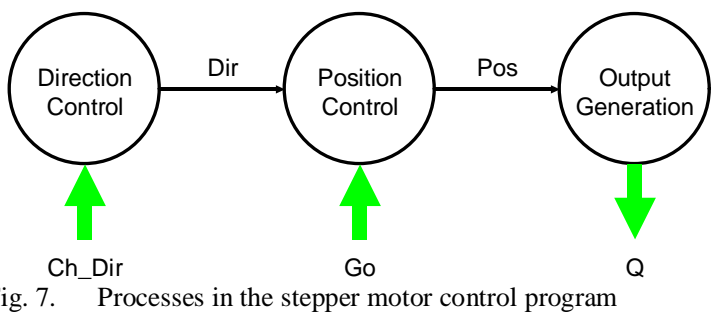

clock rate of $50 \mathrm{MHz}$ ) and the pin used for the external clock.

Lines 14-16 define constants, and then lines 20-30 define the program variables. $Q$ is the four-bit control output while G o_Sigand C hange D ir Sigare the onebit inputs, requiring corresponding variables $\mathrm{G} \circ$ and $C$ hange $D$ ir Three additional variables are required: $\mathrm{D}$ ir to indicate the current direction of rotation, $C$ hange $D$ ir_En to indicate whether a change in direction is currently enabled, and Posto indicate the current position in the output sequence. As there are four output steps Poshas a bit length of 2.

Lines 34-36 specify channel protocols which map each channel onto external pins. The particular protocols used do not implement handshaking, and so input and output are always available to the program channels. This is appropriate as the pins are connected to passive electronics - there are no external processes to synchronise with. Further details of channel protocols can be obtained from Spivey and Page (1993).

The program proper begins at line 40, and lines 42 and 44 list the external protocols and internal resources used by the program (see Section 3.4). The program code at the highest level of description consists of a two statement sequence (starting line 46): initialisation of variables using a multiple assignment (line 51), followed by a Parcontaining the three processes. Each process is of the form $W$ hile (TRU E, s)and hence loops indefinitely.

The first process (lines 57-70) implements the direction control loop, and consists of a three step sequence. The Change_Dir button is read through its channel (line 59), and then its value is inverted (line 60), as the signal conditioning (Fig. 7) generates a low signal on pin 59 when the button is pressed, and high otherwise. Finally, a compound if statement (lines 62-68) updates the values of D ir and C hange D ir En appropriately. The role of $C$ hange $D$ ir $E n$ is simply to ensure that the button is released before another change of direction can occur, as required in the problem statement. Note the use of Skip as a null else clause on line 65 .

The second process (lines 76-89) implements the position controller and has a similar structure to the first. The channel corresponding to the Go button is read (line 78) and corrected for sense (line 79). Next, if the Go button is pressed then the position is updated, according to whether the current direction is clockwise or counter-clockwise (lines 81-87). Integers are unsigned and arithmetic operates modulo $2^{\mathrm{n}}$ where $\mathrm{n}$ is the bit length of an expression, and so Pos cycles round the values $0-3$ as required.

The final process (lines 91 - 98) implements the output generation. Currently Handel does not provide latching 
of FPGA output pins, and so for this application the output must be generated every clock cycle. This process therefore uses the current value of Pos to calculate the required outputs (as given in Table 2) in a single statement.

Results. In view of the simple nature of the program, there is little need to discuss the performance of the program itself other than to say that it successfully carried out the assigned task. Of more interest are the FPGA resources needed to implement the program, and the human effort needed to design the program.

The FPGA design resulting from the program used 26 CLBs, of which 21 flip-flops were used. This is greater than the resources that might be required by an efficient solution designed at the CLB level (perhaps 8 CLBs), but seems an impressively small implementation of 60 lines of high level code. The design effort amounted to a few hours for a novice Handel programmer; with experience, the task might take an hour or less.

The section on sensor validation includes the description of a more elaborate design task for which both conventional and hardware compilation techniques were used. This presents a more complete comparison of the two approaches.

\section{SENSOR VALIDATION}

\subsection{Introduction}

This section outlines an area of control engineering research, sensor validation, and explains how FPGA and Hardware Compilation technologies are being used to enhance various aspects of the research.

\subsection{The SEVA Project}

For a number of years the Control Engineering group at Oxford has been developing the concept of the selfvalidating or SEVA sensor (Henry and Clarke, 1993). This is a device with computational power, and a digital communication capability which enables it to send and receive complex messages. Like many intelligent sensors available commercially, the SEVA sensor performs self-checking. Instead of outputting simple device-specific error codes or validity bits, it generates generic validity metrics for each measurement (Fig. 8).

The most important of these is the on-line uncertainty of each measurement. In this context uncertainty is used in the metrological sense (Kline and McClintock, 1953; ANSI, 1985) i.e. 95\% confidence limits on the measurement error. However, in the SEVA scheme the concept is extended beyond the standard definition: if a fault occurs the measurement is corrected to the best of the sensor's ability (projecting from historical data if necessary), and the uncertainty indicates the estimated quality of that estimate. Henry and Clarke (1993) de-

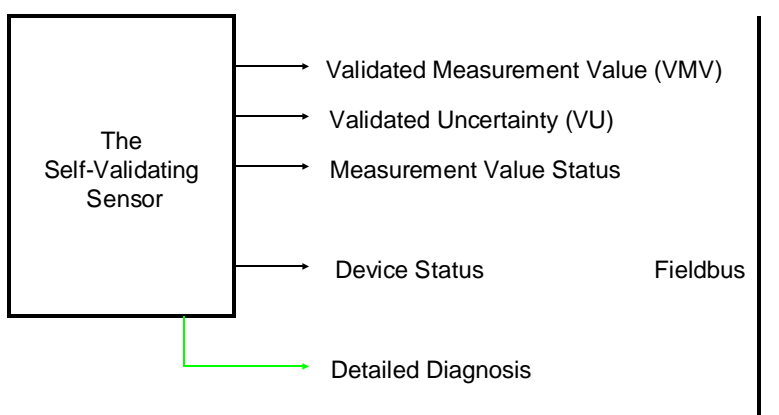

Fig. 8. Parameters generated by the Self-Validating Sensor

scribe the motivation behind the SEVA sensor concept, while Henry (1994b) presents a number of ways in which on-line uncertainty data might be used to integrate sensor validity data into various aspects of plant management.

There are several areas of active research:

- The development of prototypes of self-validating sensors. These include a coriolis meter (Henry, 1994a), thermocouple (Yang and Clarke, 1993), dissolved oxygen and pressure sensors.

- Theory and examples of the self-validating actuator, with particular reference to valves.

- Fault detection and validation of loop and process operation, assuming the availability of self-validating instrumentation.

- Advanced control using self-validating instrumentation.

\subsection{An Industrially-Focused Agenda}

SEVA is a long-range research project developing new concepts in industrial instrumentation and control based upon the emerging information technologies. It is industrially-focused, intended to develop solutions which are appropriate and acceptable to industry, not least in order to satisfy the industrial sponsors.

However, the process industries are understandably conservative. Gradual emergence from the world-wide recession has yet to result in production capacity being fully utilised, and management orthodoxy still emphasises cost-cutting rather than the taking up of new technologies. It is thus heartening that much interest has been shown in SEVA concepts, particularly by companies developing an understanding of Fieldbus technology. However, for SEVA to become an industrial reality, there is a further fundamental problem to overcome. Manufacturers do not wish to develop products until there is a market; potential users are not ready to consider the technology unless reliable products are available from multiple sources.

It is also necessary to respond to specific concerns. One issue that is frequently raised when SEVA concepts are presented to industrial engineers is that of additional complexity. How can it be demonstrated that extra 
validation features do not actually reduce overall device reliability by adding to its complexity?

These difficulties are fairly typical of any innovation proposed to both suppliers and users in industry, and there is a well-trodden route for solving them, namely the development of prototypes and demonstration systems. Careful searching may reveal applications for which the partial solutions provided by an immature technology may yet, with suitable safeguards, provide significant benefits. To some extent this entails becoming the much-maligned solution looking for a problem. This is of course a two-way exchange, as feedback from industrial experience may have a fundamental influence on the theoretical work. The development of prototypes and demonstrations, and the search for appropriate industrial applications, are thus an integral part of the research project.

\subsection{Sensor Prototypes}

The development of a SEVA sensor prototype entails interfacing a computer (normally a PC) to an industrial sensor to pick up signals relating to measurement and diagnostics. It is also possible for control signals to pass from the $\mathrm{PC}$ to the sensor, for example to activate testing circuitry.

An industrial sensor normally consists of two parts. The transducer interfaces directly with the process and generates raw data, while the transmitter (typically with a microprocessor) monitors the transducer, and converts the raw data into measurements available to the control system. When developing a SEVA prototype of a simple sensor (e.g., a thermocouple) it is possible to bypass the transmitter entirely and link the PC directly to the transducer, but for more sophisticated instruments, such as the coriolis meter, the transmitter electronics may be required to drive the transducer actively.
The PC acts as a self-validating transmitter by performing fault detection, validation, and measurement and uncertainty calculations. The net effect is that the PC and sensor together provide a concrete demonstration of how a standalone SEVA transmitter would behave if market demand were to support its development.

There are a number of reasons for basing prototypes upon existing commercial devices. Firstly, partial results (e.g., improved fault detection and compensation methods for specific instruments) have been fed back into the design of the commercial product. More important, however, is the ability to exploit the enormous design effort undertaken to develop an industrial, as opposed to laboratory, instrument. SEVA prototypes are thus more readily applicable to industrial contexts. In addition the users of the current product are more likely to be interested in demonstrations.

A disadvantage of using commercial sensors is that access to the desired signals is not readily available, as the devices were not developed with such provision in mind. The development of a sensor-specific interface to the PC is an intensive task, typically requiring several worker-months of effort, with regular liaison with the sensor design team. This work is frequently on the critical path of the entire instrument validation process, because the development (or confirmation) of diagnostic methods depends upon having access to the relevant signals so that their behaviour may be observed in a variety of faulty and non-faulty conditions.

For example, Fig. 9 illustrates the interfacing circuitry required for the coriolis meter validation system. Three signals provide raw measurement data in the form of frequency pulses, while seven signals are used for fault detection purposes. A total of sixteen digital output lines are used to manipulate the transmitter circuitry to implement a number of tests and control operations. In

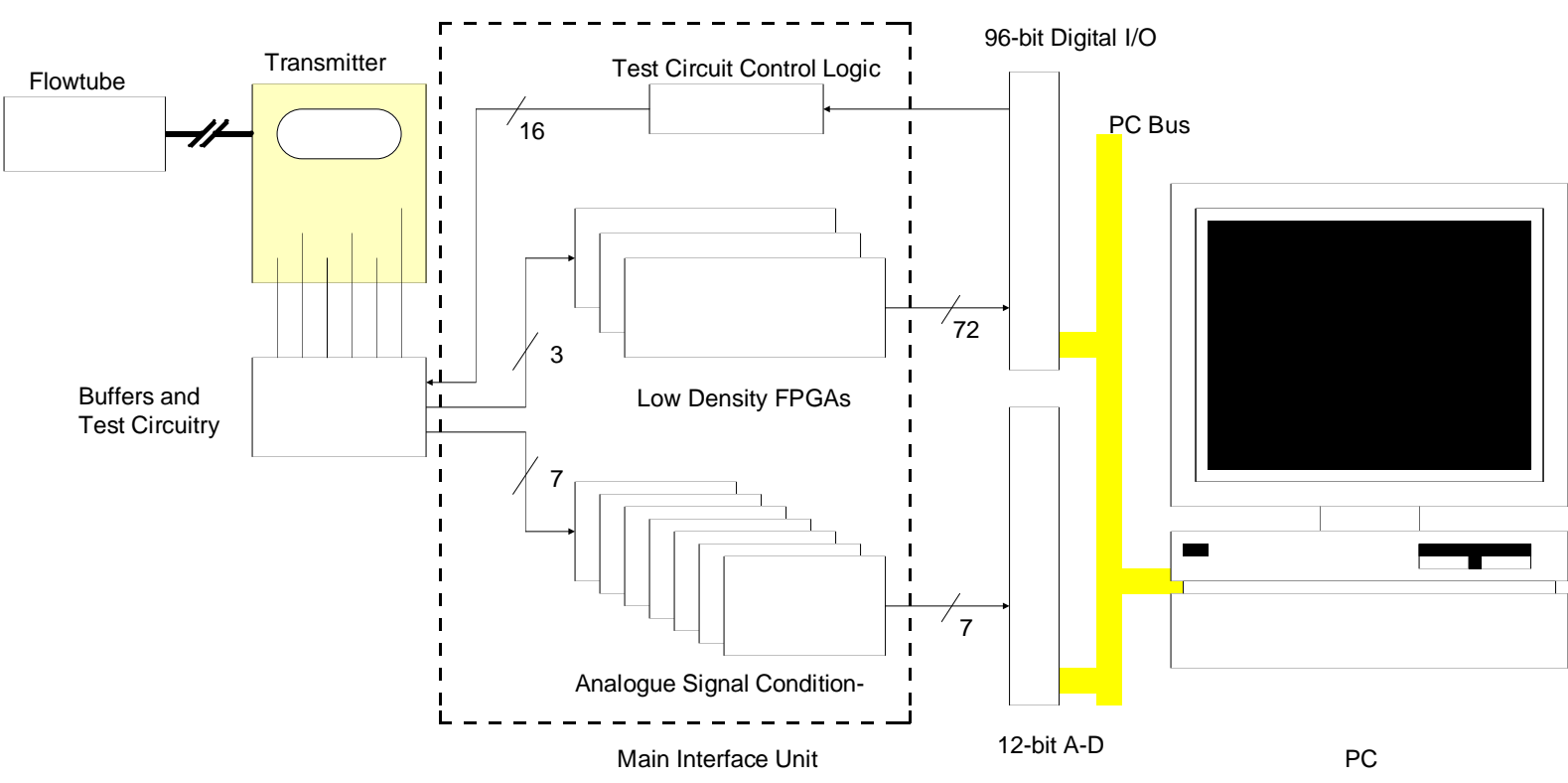

Fig. 9. Architecture of coriolis meter validation system 
order to supply the measurement data to the PC with sufficient precision, an FPGA was used on each channel as described later.

The prototypes developed so far have successfully demonstrated equivalent or improved accuracy over their commercial counterparts, provided innovative device-specific fault detection techniques, and demonstrated the concept of generating on-line uncertainty in faulty and non-faulty conditions (Henry, 1994a; Yang and Clarke, 1993).

\subsection{Industrial Demonstrations}

A number of demonstrations of SEVA prototypes have taken or are taking place in a variety of applications drawn from the food, paint, petrochemical and power industries. When a system is to be used in these contexts, safety and reliability requirements are more stringent. For example, one industrial trial took place in a Zone 1 area. Although the commercial transmitter itself was certified for Zone 1, the modified device was not, and so additional engineering was required to upgrade the demonstration system.

A further difficulty concerns the interfacing of a prototype system to an existing control system. This is necessary to move beyond the passive collection of data and towards generating process responses to validation data generated by a SEVA prototype. Of course, most industrial control systems still operate using the univalued, unidirectional 4-20mA standard. This presents problems when trying to introduce multiple streams of data (i.e. measurement plus validity). Many control computers are capable of handling other forms of input, e.g., binary alarm indicators, RS-232, RS-423, IEEE1118, and in due course Fieldbus. Communications must thus be engineered on a case-by-case basis.

\subsection{The Valcard Project}

The last sections have described some of the difficulties of developing and demonstrating SEVA prototypes. Many of these could be ameliorated considerably by the application of hardware compilation techniques, through rapid and reliable prototyping of interfaces.

A research project called Valcard has been undertaken jointly by the Control Engineering Group and the Computing Laboratory at Oxford. The purpose of the project is to develop a 'validation card' suitable for prototyping self-validating instruments. The card will include a transputer and FPGAs, together with flexible i/o devices (such as sigma-delta converters (Boser and Wooley, 1988)). Hardware compilation techniques will be used to configure the card appropriately for each application. For example, Fig. 10 illustrates how the valcard might interface to the coriolis transmitter. Although some signal conditioning will be required in almost every application, it should be minimal, and readily fitted onto standardised daughter boards.

The inclusion of a transputer means that measurement, fault detection and validation calculations can all occur on the board itself, rather than in the host computer. This offers a number of practical benefits such as the separation of host and instrument software, the use of non-PC host computers, and the ability to house several validation cards in a single host.

Although the project is in its early stages, the results so far are impressive, and indicate that hardware compilation can provide a massive increase in design productivity. For example, in the coriolis interfacing illustrated in Fig. 9, low-density FPGAs were used to provide highly accurate measurements of frequency signals generated by the transmitter. Conventional CAD techniques were used to configure the FPGAs. Design and debugging took several weeks. As part of the Valcard project, the functionality was replicated in Handel in less than an hour (Page and Randall, 1994). A number of technical difficulties had to be resolved

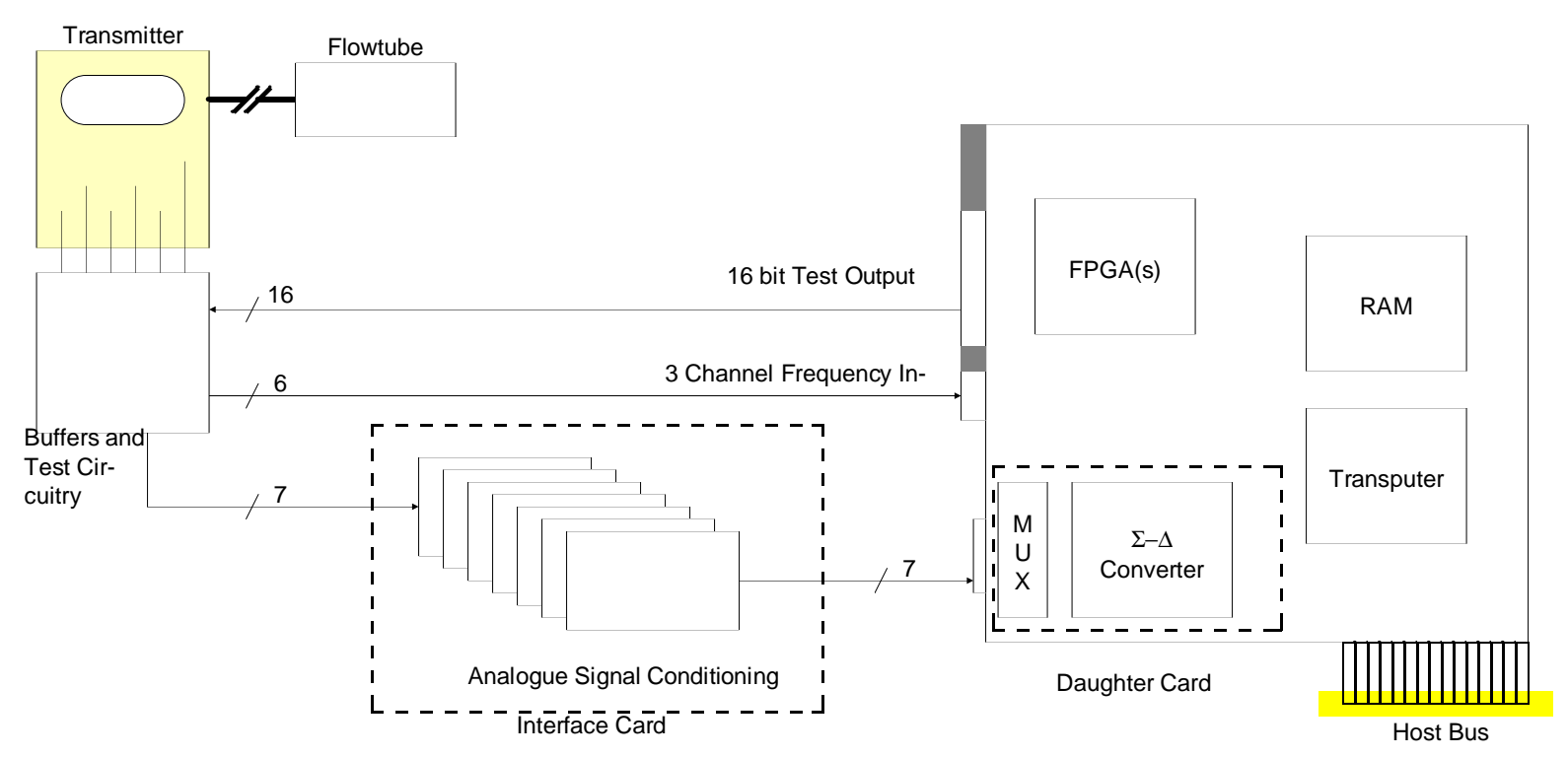

Fig. 10. Proposed Valcard implementation of coriolis validation system 
before the FPGAs could be programmed, some of which entailed extensions to the Handel compiler, but these have now been solved. The implementation of even remotely similar functionality is now available in a very rapid and reliable technology.

The project experience has not merely been a matter of speeding up tasks that were previously achievable. In validation work on a dissolved-oxygen meter, a PC card was developed which included a sigma-delta ADC (Analog Devices, 1992) controlled by an FPGA. The high measurement precision delivered by the card enabled the demonstration and verification of a novel fault detection and correction method which would have been impossible using the conventional instrumentation. Again, with the FPGA configuration available in a high-level form, it is readily modified for use in different applications.

Finally, the use of hardware technology has suggested new lines of research. With the provision of local power and a communications capability, the valcard can become independent of any host computer, adopting the attributes of a universal transmitter. This possibility suggests the further exploration of a number of issues:

- A variety of communication protocols can be implemented in Handel, either to match the requirements of a particular industrial application, or to assist in the development of SEVA-related protocols (e.g. for Fieldbus). A body of work has been carried out on the formal specification of communication protocols (e.g. (Neumann, 1994)); this could be extended using hardware compilation techniques to provide automated generation of an FPGA configuration to match the specification of a communication protocol.

- A standalone card provides a natural focus for the development of a general software architecture for sensor validation, as there is a high degree of functional overlap between any two devices. The development of generic software in occam and Handel can be assisted by the use of formal methods to ensure high-integrity code. Such a development would be an appropriate response to concerns about the overall reliability of the SEVA sensor despite enhanced functionality, and raise the prospect of rapid and reliable prototyping of the software and hardware for a new instrument.

Although the Valcard concept was developed in the context of sensor validation, it may have wider uses. The inclusion of DACs, for example, would create a flexible and reliably-configurable general-purpose card for control applications. Interest has already been shown by other research groups in using the valcard as an intelligent communication node and as an intelligent controller for variable-speed drives for Fieldbus.

\section{CONCLUSIONS}

This paper began with tutorial introductions to the concepts of FPGAs and hardware compilation, which included a simple demonstration of a stepper motor control program. This was followed by a description of the sensor validation research programme and of the several benefits achieved and expected from using hardware compilation techniques. Clearly the SEVA project is not unique in being able to benefit from hardware compilation, and it is hoped that this paper will draw the attention of other likely beneficiaries in the control engineering community to this extremely powerful tool.

In conclusion, some reflections are given on the complex relationship between technology and research, particularly in the context of control engineering.

The process which generates technology is termed innovation, and, as Kline (1985) points out, innovation is not a linear process. The traditional 'linear' view is that research leads on to development, production and finally the marketing of a product. Kline argues that this model is inadequate, and presents his own 'chainlinked' model. In Kline's view, research generates 'knowledge', which is used, perhaps only after many years delay, in any or all phases of technological development. Kline points out that far from being a linear process, numerous feedback loops occur during innovation, including the assistance that technology has on subsequent research.

Technology assists research in at least three ways:

- it speeds up tasks that were previously possible, but tedious - for example, automated calculation rather than by hand, or the use of hardware compilation rather than CAD configuration of an FPGA.

- it provides existing research with results not previously obtainable - for example, the output from the Hubble telescope, or the results of using a sigma-delta ADC on the dissolved oxygen sensor.

- it can inspire entirely new fields of research - for example, the existence of image storage and transmission technologies has triggered research into image compression, and the existence of FPGAs led directly to the research initiative on hardware compilation and hybrid architectures.

The relationship between knowledge generation and technology is thus complex, and interaction between the two takes place at every level of research activity. This is true of control engineering, which is concerned with the modelling and control (i.e. obtaining desired outputs) of systems, particularly those mechanical, industrial, technological and biological systems which are of practical concern to human kind. Technology 
interacts not only with how its problems are defined and solved, but also how it is applied in practical domains.

The most obvious examples are where new technology directly introduces control methods for the first time for example, digital controllers have made widespread the use of auto-tuning in PID loops. However, it is also possible for technology to modify the system under control, requiring new control strategies to be devised.

An example of the latter is provided by developments in process fault detection, arguably a subset, or at least related to, control theory. The prevailing methodology is known as Fault Detection and Isolation (FDI (Patton, 1994)). A key premise is that, viewed from the central computer in which FDI takes place, it is not in general possible to distinguish between a sensor fault and a change in the process, and so a process model must be developed exploiting analytical redundancy relationships between measurements to spot inconsistencies.

As argued by Henry and Wood (1992), this premise is challenged by the widespread introduction throughout the process industries of digital instrumentation and field communications. Increasing numbers of 'intelligent' sensors and actuators containing rudimentary diagnostics are being installed, certainly at a faster rate than those monitored by FDI schemes; it is highly unlikely that this trend will be reversed. The environment these methods were designed to be applied in is evolving more rapidly than the methods themselves.

Of course, FDI theory remains valid, but it may become increasingly unattractive in a domain which in part inspired it, because technological developments are providing more economic alternatives. This argument does not hold for other application domains for FDI, such as aerospace (Patton, 1991), and indeed technological advances (micro-sensors) and socio-economic pressures are introducing new application domains, such as the automobile (Gertler and Costin, 1994).

However, it is 'information technology' or 'knowledge engineering' which is having the most profound and direct impact upon control research itself, as on many other subjects. One effect is to tighten the coupling between the generation of knowledge and its application (i.e., 'research' and 'technology') through the provision of powerful software environments. This generates greater positive feedback and hence productivity.

For example, it is claimed that a particular computer algebra package is used by a high proportion of the mathematical research community in the United States, and that the results of the latest research are ploughed back into the program at regular intervals. The control engineering community has had a similar if less dramatic experience through tools such as MATLAB. One worker's research readily becomes another's technol- ogy. This paper has illustrated how an early result of the hardware compilation research programme - the Handel compiler - has already proved to be effective technology for furthering the aims of the SEVA project.

While information technology is benefiting many subjects, it is particularly influential in control engineering. At least with the physical sciences there is an external reality about which 'pure' knowledge can be gathered, which is typically beyond the descriptive power of existing computer languages. The 'laws' of physics must be changed and extended to fit the observed facts. Control engineering by contrast is about description and method - language and algorithm, the very stuff of information technology. The system descriptions used in control - for example state space and transfer function - are relatively few and readily captured by existing automation tools. Indeed, rather than actively creating new system descriptions, control engineers are embracing other paradigms developed within IT itself - fuzzy logic, neural nets, Petri-nets, and the various formulae of artificial intelligence, etc, to develop descriptions of and solutions to control problems which are not amenable to its traditional approaches.

Indeed, it may be argued that the distinction between research (i.e., generating knowledge), acquiring knowledge (i.e., understanding the research of others) and applying technology may rapidly become little more than the following:

- Research: develop, write and test a procedure body;

- Acquiring knowledge: read a procedure body;

- Applying technology: read the procedure heading and use it.

As suggested earlier in this paper, even the distinction between hardware and software may fade.

The information technology revolution is as yet in its infancy, and is already having a profound effect upon all aspects of control - how it is formulated, how its problems are solved, and how it is applied. At the very least, it is going to dictate the control agenda for the foreseeable future, but it is possible to speculate further. Will control engineering, once a thriving independent community, soon become just another suburb of the rapidly-expanding information technology metropolis?

\section{ACKNOWLEDGEMENTS}

The author is grateful for the tireless assistance of Ian Page, the creator of Handel, Geoff Randall, working on the Valcard project, and Jeremy Bowles, who first introduced the author to FPGAs. This work was partly funded by SERC grant no GR/J44636 (Valcard) and the Foxboro Company. 


\section{REFERENCES}

Abrial, J.-R. (1985). Programming as a mathematical exercise, in Mathematical Logic and Programming Languages, C.A.R. Hoare and J. C. Shepherdson eds, Prentice-Hall International, Hemel Hemptstead, UK.

Aho, A. V. and J. D. Ullman (1977). Principles of Compiler Design, Addison-Wesley, Reading, Massachusetts, USA.

Altera (1993). Max 7000 Data Book. Altera Corporation, San Jose, California, USA.

AMD (1991). Palasm 4 User's Manual. Advanced Micro Devices, Inc., Sunnyvale, California, USA.

Analog Devices (1992). AD7711 - LCCMOS signal conditioning ADC with RTD excitation currents. Analogue Devices, Limerick, Ireland.

ANSI (1985). Measurement Uncertainty. ANSI/ASME PTC 19.1-1985.

Atmel (1993). Configurable Logic Design and Application Book. Atmel Corporation, San Jose, California, USA.

Boser, B. E. and B. A. Wooley (1988). The design of sigma-delta modulation analog-to-digital converters. IEEE J. Solid-State Circuits, vol SC-23, pp 1298-1308.

Data I/O (1993). Abel Design Software User Manual. Data I/O Corporation, Redmond, Washington, USA.

FutureNet (1987). Dash Schematic Designer. FutureNet Corporation, Chatsworth, California, USA.

Gertler, J. and M. Costin (1994). Model-Based Diagnosis of Automotive Engines - Case Study on a Physical Vehicle. IFAC/IMACS Safeprocess Symposium, Espoo.

Henry, M. P. and D. W. Clarke (1993). The Self-Validating Sensor: Rationale, Definitions and Examples. Control Engineering Practice, 1, 585.

Henry, M. P. and G. G. Wood (1992). The implications of digital communications on sensor validation. IFAC Symposium on On-line Fault Detection and Supervision in the Chemical Process Industries, Newark.

Henry, M. P. (1994a). A SEVA Sensor - The Coriolis Mass Flow Meter. IFAC/IMACS Safeprocess Symposium, Espoo.

Henry, M. P. (1994b). The Integration of Fault Detection within Plant-wide Data Quality Management. IFAC/IMACS Safeprocess Symposium, Espoo.

Hoare, C. A. R. (1985). Communicating Sequential Processes. Prentice Hall International, Hemel Hempsted, UK.

Hoare, C. A. R. and I. Page (1994). Hardware and Software: The Closing Gap. Transputer Communications, Wiley, 2(2), June 1994, 69-90.

IEEE (1994). VHDL revised standard (VHDL 10761993).

Inmos (1988a). occam2 Reference Manual. Prentice Hall International, Hemel Hemptstead, UK.

Inmos (1988b). Transputer Reference Manual. Prentice Hall International, Hemel Hemptstead, UK.

Jones, G. and M. Goldsmith (1988). Programming in occam2. Prentice Hall, Hemel Hempstead, UK.

Kline, S. J. and F. A. McClintock (1953). Describing uncertainties in single sample experiments. Mech. Eng., pp3-8.

Kline, S. J. (1985). Innovation is not a linear process. Research Management, Vol 28, Part 4, pp 36-45.

Knapp, S. (1990). Accelerate FPGA Macros with One Hot Approach, Electronic Design, Sept 13, 1990.

Lattice (1994). Lattice Data Book 1994. Lattice Semiconductor Corp., Hillsboro, Oregon USA.

Milner, R., M. Tofte and R. Harper (1990). The Definition of Standard ML. The MIT Press, Cambridge, Massachusetts, USA.

Neumann, P. (1994). Implementation support for Communication Systems. Research summary, Institut für Automation und Kommunication e. V. Magdeburg, Barleben, Germany.

Omation (1988). Schema II Reference Manual. Omation Inc., Richardson, Texas, USA.

Oxford Parallel (1994). The Oxford Parallel FPGA design. Internal report, Oxford Parallel, Oxford, UK.

Page, I. (1993). Automatic Systems Synthesis: A Case Study. Internal Report, Oxford University Computer Laboratory.

Page, I. (1994). Parameterised Processor Generation. In More FPGAs, W. Moore and W. Luk (eds), Abingdon EE\&CS Books, Oxford.

Page, I. and W. Luk (1991). Compiling occam into Field-Programmable Gate Arrays. In FPGAs, W. Moore and W. Luk (eds), Abingdon EE\&CS Books, Oxford.

Page, I. and G. Randall (1994). Using Handel To Accelerate Systems Development, IEE Colloquium On "Fast Prototyping Of IC Designs", London.

Patton R. J. (1991). Fault Detection and Diagnosis in aerospace systems using analytical redundancy. IEE Computing and Control Journal, Vol 2, 127-136.

Patton, R. J. (1994). Robust Model-Based Fault Diagnosis: The State of the Art. IFAC/IMACS Safeprocess Symposium, Espoo.

Schlag D. F., P. K. Chan and J. Kong (1990). An Empirical Study of the Performance of Multilevel Logic Minimisation Tools for a Field-Programmable Gate Array Technology, University of California at Santa Cruz, Computer Science Department Report UCSC-CRL-90-60.

Spivey, M. and I. Page (1993). How to design hardware with Handel. Internal Report, Oxford University Computer Laboratory.

Viewlogic (1991). Workview Manual. Viewlogic Systems, Inc., Malboro Massachusetts, USA.

Vonderschmit, B (1994). Programmable Logic in the Year 2000. Xcell Issue 13, Xilinx Inc., San Jose, California, USA.

Xilinx (1993). XACT Development System User Guide. Xilinx Inc., San Jose, California, USA.

Xilinx (1994). The Programmable Logic Data Book. Xilinx Inc., San Jose, California, USA.

Yang, J. C-Y. and D. W. Clarke (1993). A self-validating thermocouple. Internal Report, Department of Engineering Science, University of Oxford. 


\section{Appendix 1: Stepper Motor Control Program}

( $*$ This is the fullSM L description of the stepperm otor controller

M .H enry 1stAugust $94 *)$

(* Architectural issues *)

6: fipga_controlclock_pad := "P13"; (* use extemalclock viapin 13 *)

: fpga_control.fpga_chip := "2018PC 84-50"; (* 50M H z 2018 w ith 84 extemalpins *)

8: fipga_controlffipga type = fipga_controlx ilin $\times 2000$;

fpga_controlfinish_pad = '"';

: fpga_controlnotEmor pad := '"';

(* C onstants *)

14: valN O_Steps =

15: valW_Pos = ilog $2 \mathrm{~N}$ o_Steps

16: valNo_C_Lines $=4$

(*um ber of stepperpositions *)

(* w idth of position register *)

$17:$

18: (* C hannels *)

19:

20: valG o_Sig = Chan 1 'G o_Sig" (* input from go button *)

21: valChange_D ir_Sig = Chan 1 "Change_D ir_Sig" (*inputfrom change dirbutton *)

2: valQ_Sig = Chan N O_C_Lines 'Q_Sig"; (* output to $\mathrm{m}$ otorcontrol*)

4: (* Variables *)

25:

26: valGo = Int1 'G O" (* holdsgo input*)

27: valChange_D ir = Int1 "C hange_D ir" (* holdschangedirection input*)

28: valChange_D ir_En = Int1 "Change_D ir_En" (* change direction enabled? *)

29: vald ir = Int1 "D ir" (* direction, $1=$ clockw ise *)

30: valPos = IntW_Pos "Pos" (* position in steppercycle *)

31:

32: (* fullchanneldefinitions: these m ap channels onto FPG A pins *)

33:

34: valG o_Sig_C PC = (C PC_N hPortin 10 [ "P59"], Input Go_Sig);

5: valCD S_C PC = (C PC_N hPortIn 10 [ "P56"], Input Change_D ir_Sig);

6: valQ_C $\overline{P C}=$ (C PC_N hPorto ut10 [ "P 81", "P83", "P79", "P 82"], O utputQ_Sig);

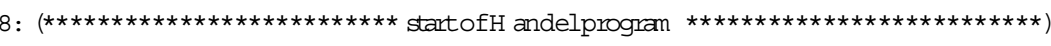

prog $:=\mathrm{H}$ andel (

: [G o_Sig_CPC , CD S_C PC , __C PC ], （* program channels *)

$43:$

44: [G o,C Change_D ir, D ir, Pos, C hange_D ir_En ], (* program variables *)

$45:$

6: Seq [ (* program execution consists of an in itialisation follow ed by

47: three parallelprocesses, each of w hich loops indefinitely *)

(* initialisation *)

[POS,D ir, Change_D ir_En] $=[\mathrm{C} 0, \mathrm{C} \quad 0, \mathrm{TRU} \mathrm{E}]$,

( parallelprocesses *)

Par [

W hile (TRU E,

Seq [

(* direction controlloop *)

Change_D ir_Sig ?? C hange_D ir, (* get inputsignal *)

Change_D ir $=\sim$ Change_D ir, (* inputhas inverted sense *)

If (C hange D ir, (* update change_dir, change_dir_en *)

If (C hange D ir En,

[D ir, C hange_ $\bar{D}$ ir_En $]=[\sim \mathrm{D}$ ir, FALSE $]$,

Skip

),

Change $\mathrm{D}$ ir En $=\mathrm{TRUE}$

)

(* end of com pound if*)

(* end of sequence *)

), (* end ofdirection controlloop *) 
74: (* StepperM otor C ontrol Program Listing continued ... *)

$75:$

76: W hile (TRU E,

Seq [

Go_Sig ?? Go,

$\mathrm{Go}:=\sim \mathrm{Go}$,

If (Go,

If (D ir,

POS $=$ POS $+\mathrm{C} 1$,

POS $=$ POS $-\mathrm{C} 1$

),

Skip

)

]

)

W hile (TRU E,

Q_Sig!!(
(* position control loop *)

(* a sequence of steps *)

(* get input signal *)

(* inputhas inverted sense *)

(* m odify position? *)

(* yes - w hich direction? *)

(* clockw ise - increm ent*)

(* counter-clockw ise-decrem ent*)

(* no - do nothing *)

(* end ofcom pound if *)

(* end of sequence *)

(* end ofposition control loop *)

(* outputgeneration loop *)

(* bit-w ise construction of output *)

((POsBIT 1) EXOR (PosB IT 0)) (* bito *)

$\wedge \sim($ POsB IT 1) (* bit1 *)

^ ((POsBIT 1) EXOR (POsBIT 0)) (* bit2 *)

^ (POsB IT 1) (* bit3*)

)

)

(* end ofoutputgeneration loop *)

(* end of parallelprocesses *)

(* end ofprogram sequence *)

(* end ofprogram *)

102:);

103:

104: (************************** end of $\mathrm{H}$ andelprogram 